\title{
Recommending Education Materials for Diabetic Questions Using Information Retrieval Approaches
}

Yuqun Zeng ${ }^{1,2,3 *}$; Xusheng Liu ${ }^{1 *}$; Yanshan Wang ${ }^{2}$; Feichen Shen $^{2} ;$ Sijia Liu ${ }^{2,4}$; Majid Rastegar-Mojarad² ${ }^{2}$ Liwei Wang $^{2}$; Hongfang Liu $^{2}$

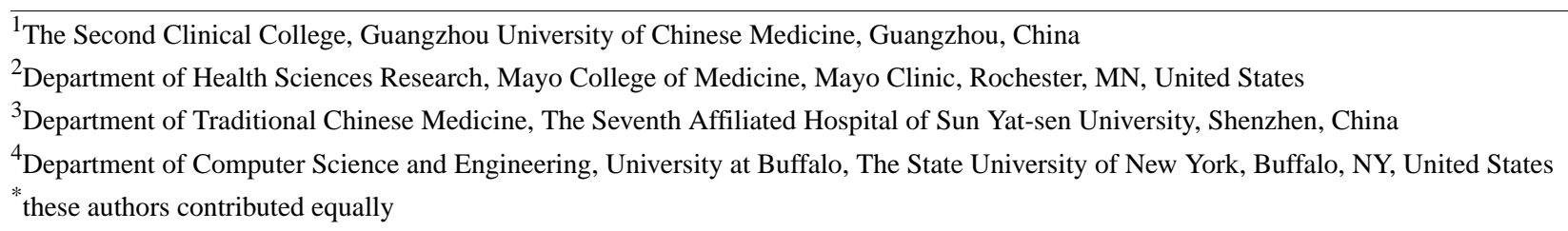

Corresponding Author:

Hongfang Liu

Department of Health Sciences Research

Mayo College of Medicine

Mayo Clinic

200 First Street SW

Rochester, MN, 55905

United States

Phone: 15072930057

Fax: 15072841516

Email: Liu.Hongfang@mayo.edu

\section{Abstract}

Background: Self-management is crucial to diabetes care and providing expert-vetted content for answering patients' questions is crucial in facilitating patient self-management.

Objective: The aim is to investigate the use of information retrieval techniques in recommending patient education materials for diabetic questions of patients.

Methods: We compared two retrieval algorithms, one based on Latent Dirichlet Allocation topic modeling (topic modeling-based model) and one based on semantic group (semantic group-based model), with the baseline retrieval models, vector space model (VSM), in recommending diabetic patient education materials to diabetic questions posted on the TuDiabetes forum. The evaluation was based on a gold standard dataset consisting of 50 randomly selected diabetic questions where the relevancy of diabetic education materials to the questions was manually assigned by two experts. The performance was assessed using precision of top-ranked documents.

Results: We retrieved 7510 diabetic questions on the forum and 144 diabetic patient educational materials from the patient education database at Mayo Clinic. The mapping rate of words in each corpus mapped to the Unified Medical Language System (UMLS) was significantly different $(P<.001)$. The topic modeling-based model outperformed the other retrieval algorithms. For example, for the top-retrieved document, the precision of the topic modeling-based, semantic group-based, and VSM models was $67.0 \%, 62.8 \%$, and $54.3 \%$, respectively.

Conclusions: This study demonstrated that topic modeling can mitigate the vocabulary difference and it achieved the best performance in recommending education materials for answering patients' questions. One direction for future work is to assess the generalizability of our findings and to extend our study to other disease areas, other patient education material resources, and online forums.

(J Med Internet Res 2017;19(10):e342) doi: 10.2196/jmir.7754

\section{KEYWORDS}

education materials; patients; questions; recommendation; information retrieval 


\section{Introduction}

Diabetes is a chronic metabolic disease currently affecting almost 415 million patients worldwide with an estimation of this reaching 642 million by the year 2040 [1]. Having diabetes is associated with substantially higher lifetime medical expenditures despite being associated with reduced life expectancy [2]. Optimal control of diabetes requires a high degree of self-management where individuals have the necessary knowledge, skill, and ability for diabetes self-care [3]. Self-management consists of a complex and dynamic set of processes and is deeply embedded in each patient's unique situation [4]. Meeting the information needs of each patient is crucial in facilitating self-management.

Patients' self-learning is an important component of self-management. For example, through self-learning modules, patients can gain more knowledge and be more knowledgeable about practice interventions regarding foot care, which is a widely neglected part of diabetes management [5]. Meanwhile, the Internet has become an important source of self-learning for patients. Many online health communities and forums have emerged as popular platforms for patients to ask questions and share information. However, the quality of health information on the Internet is highly variable [6]. It is crucial to provide expert-vetted information to patients. At the same time, there is an abundant supply of expert-vetted patient education resources that aim to help diabetic patients improve their diabetes self-management [7-9]; however, it is quite challenging for patients without a medical background to find relevant educational materials. A system that can automatically recommend such resources to patients based on their questions in an online forum would be one way to provide relevant expert-vetted education materials.

Retrieving relevant education materials for given questions can be regarded as an information retrieval task. Information retrieval refers to the task of retrieving information of any type from a collection of documents related to search queries. One classic information retrieval approach is based on keyword matching (ie, Boolean model) [10], where documents are represented as a set of terms and queries are represented as Boolean expressions. Another popular information retrieval approach is the ranking model. Unlike the Boolean model where terms are equally weighted, the ranking model ranks the result list in terms of relevance of documents with respect to an information need expressed in the query [10]. Ranking is usually to compute numeric scores of query/document pairs where numerous scoring algorithms have been used. For example, the vector space model (VSM) computes the similarity between a query vector and a document vector, where terms can be weighted using a term frequency-inverse document frequency (TF-IDF) model $[11,12]$. One common idea of information seeking is to come up with good queries by thinking of words that would likely appear in a relevant document. The language models directly model such ideas where a document is a good match to a query if the document is likely to generate such a query. For a query, the probabilistic language model approach computes a probabilistic language model and ranks documents based on the probability of the model generating the query. Semantic searching intends to improve searches by understanding the semantics in queries and document collections. Concept mapping is popularly used in semantic searches where keywords are mapped to concepts captured in terminological resources. In general English, WordNet is a popular terminology resource where terms are grouped into sets of synonyms according to their meanings and organized into hierarchies based on their semantic relations [13].

Recently, topic modeling, which discovers abstract topics in document collections, has become a frequently used technique in text mining. The most common topic modeling approach is Latent Dirichlet Allocation (LDA), which allows documents to have a mixture of topics. For example, Wang and Blei [14] used topic modeling to generate an interpretable latent structure for users and items, which can provide recommendations about both existing and newly published scientific articles. In information retrieval, topic modeling can be effective in enabling the incorporation of hidden semantics [15].

In the clinical domain, there are many information retrieval applications [16], including clinical decision support. For example, InfoRetriever was designed for family medicine providers to practice evidence-based medicine [17]. Information retrieval technology is also popularly used in patient education applications, such as the PERSIVAL system, which is based on individual patient records and provides personalized access to a distributed patient care digital library by retrieving and summarizing relevant education materials.

Here, we propose a system that leverages the latest information retrieval techniques, which recommends patient education materials for questions asked by patients online. The system aims to provide expert-vetted, patient-faced information to patients. A similar system has been proposed by Kandula et al [18] where, instead of patients questions, their system recommended relevant education materials based on medical records. In this study, we investigated the use of state-of-the-art information retrieval approaches to recommend diabetes education materials for questions available in an online diabetes forum.

\section{Methods}

An overview of our workflow of this study is presented in Figure 1. We designed a recommendation system using three retrieval models, including a topic modeling-based model, a semantic group-based model, and a VSM. To evaluate the performance of each model in the system, we assembled a gold standard dataset created manually for a randomly sampled subset of questions. 
Figure 1. The workflow of this study.

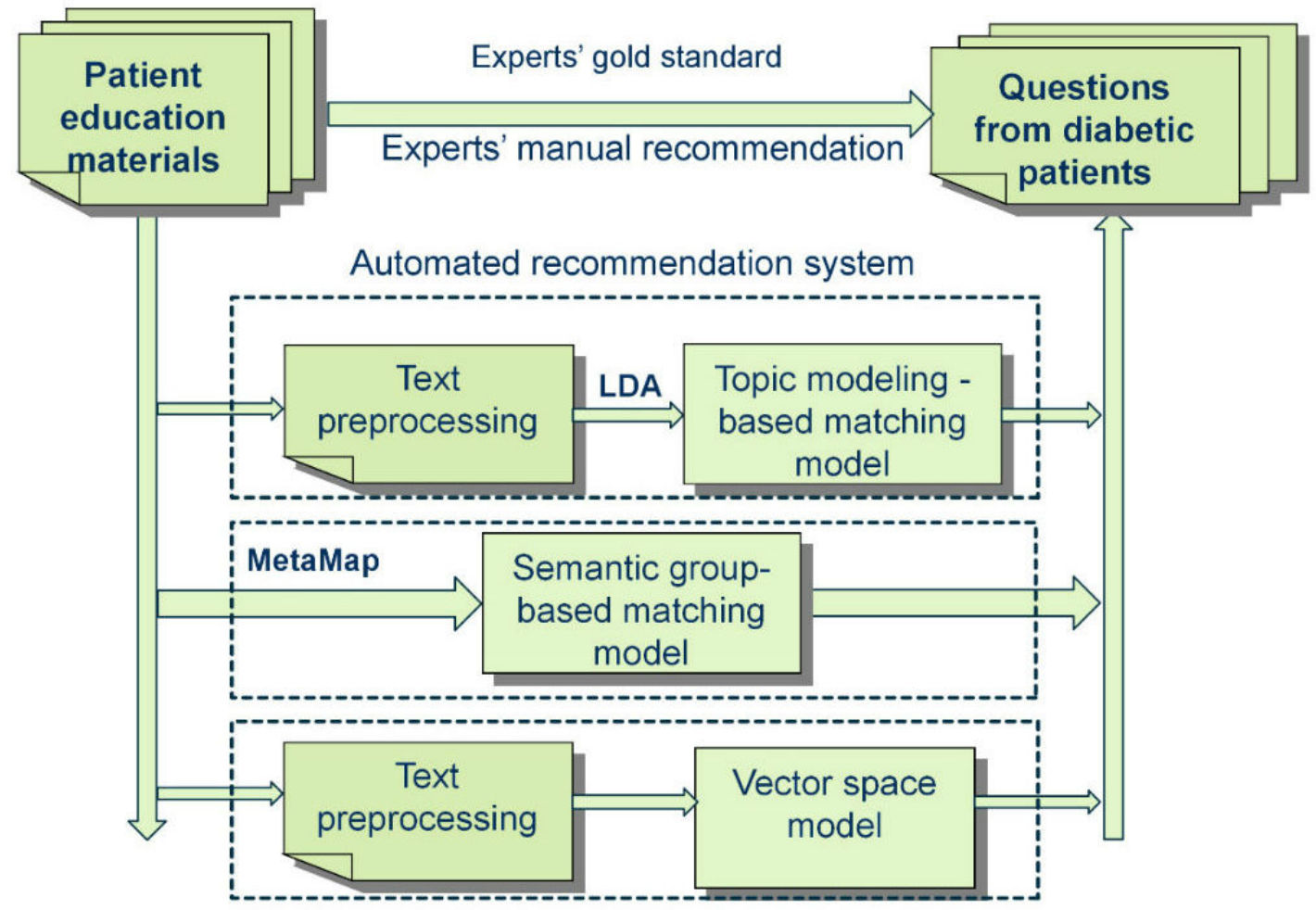

\section{Materials}

The materials used for our study included a corpus of patient educational materials for diabetic patients retrieved from Mayo Clinic's patient education database and a corpus of questions retrieved from a diabetic forum. There were more than 7400 high-quality, expert-reviewed, and outcome-based patient education materials available in the Mayo Clinic's Database of Approved Patient Education Materials, which are indexed using disease concepts. We retrieved all diabetes-related education materials, a total of 144 documents, in PDF format and used Apache Tika, a content analysis toolkit [19], to transform the PDF format to plain text and form the patient educational materials corpus. We chose a popular diabetic online forum, the TuDiabetes forum [20], to retrieve questions asked by diabetic patients. There are more than 43,000 forum users who post questions, provide answers or comments, participate in discussions, and share experiences. Questions in the forum have been categorized into 12 categories. We gathered a total of 7510 diabetic questions from the website; for each question, the corresponding title, content, and category were extracted and formed into the corpus of questions from diabetic patients.

\section{Tools}

We used the Unified Medical Language System (UMLS) from the US National Library of Medicine (NLM) and the associated concept-mapping tool, MetaMap, to represent and extract clinical concepts from the corpora. The UMLS is a comprehensive resource for clinical concepts, which integrates more than 2 million names for some 900,000 concepts from more than 60 families of biomedical vocabularies, as well as 12 million relations among these concepts [21]. Each clinical concept is assigned a concept unique identifier. The UMLS arranges clinical concepts into 134 semantic types. These semantic types are further grouped into 15 semantic groups. The MetaMap tool is a configurable app developed by NLM to map biomedical text to the UMLS Metathesaurus.

We used the LDA topic model with JGibbLDA software [22] to classify the patient education materials. LDA topic modeling is a common method that generates a high underlying set of topic probabilities with an infinite mixture based on a three-level hierarchical Bayesian model [23]. The statistical analysis was performed using $\mathrm{R}$ [24]. The attribute proportion data were analyzed using chi-square tests. We also used Cytoscape software version 3.4 to visualize the networks generated in different models [25].

\section{Information Retrieval Algorithms}

We compared three algorithms for recommending patient education materials for matching questions: (1) a VSM model as the baseline model using scikit-learn 0.18 .0 package [26], (2) a topic modeling-based matching model motivated by Kandula et al [18] using topic modeling for matching patient educational material to patient's clinic notes, and (3) a semantic group-based matching model that considered each semantic group as a topic in the patient educational materials corpus, the detail processing in Figure 2. See Multimedia Appendix 1 for the weight calculations for the topic modeling-based and semantic group-based models. 
Figure 2. The workflow of the semantic group-based model. CHEM: chemicals and drugs; CONC: concepts and ideas; CUI: concept unique identifier; DISO: disease; QDP: questions from diabetic patients; PEM: patient educational materials.

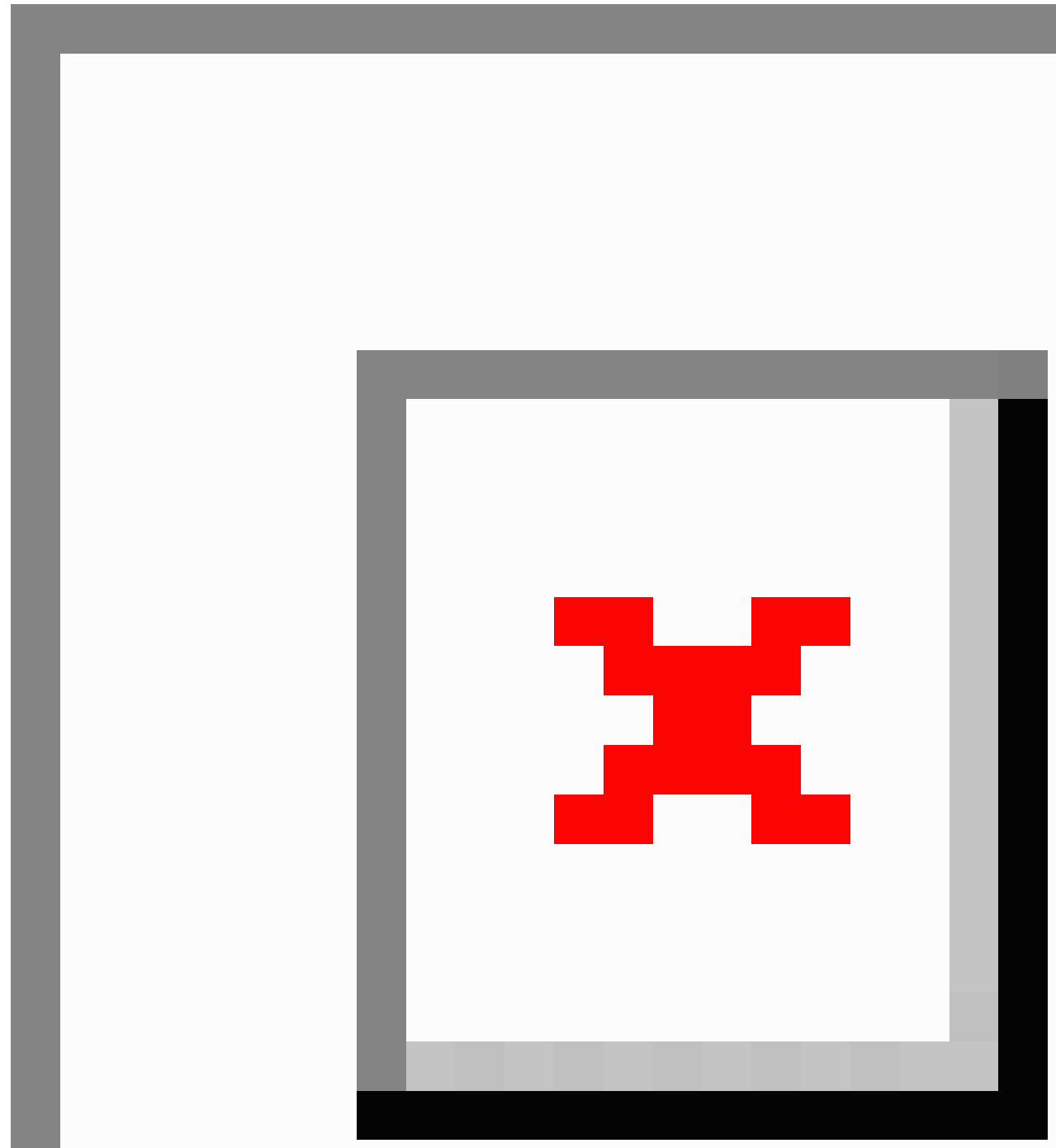

\section{Gold Standard and Evaluation}

To compare the performance, we randomly selected 50 questions and assembled a gold standard dataset based on manual review with the agreement of two experts. Specifically, for the pairing of question $q$ and education material document $d$, we manually assigned a score in the range of 0 to 2 to indicate if $\mathrm{d}$ was relevant to q, where 0 indicated no relevance, 1 partial relevance, and 2 most relevance. Two medical experts performed the task. The weighted Cohen kappa value was calculated to determine interannotator agreement. A gold standard was then created based on the consensus of the two experts. The precision of the top $k$ retrieved documents was used to evaluate the performance of the models, defined in the following:
Precision $(\mathrm{k})=($ number of relevant documents $) / \mathrm{k}$ where a partial relevance document was counted as 0.5 .

\section{Results}

\section{Overall Statistics}

As shown in Table 1, the mean document length (word count) was 968 (SD 115) and 110 (SD 36) for patient educational materials and questions from diabetic patients, respectively. The UMLS mapping rate (the ratio of words that can be mapped to UMLS concepts) for patient educational materials was significantly higher than questions from diabetic patients $(P<.001)$ with more unique concepts in questions from diabetic 
patients than in patient educational materials. The unique word count in questions from diabetic patients was 41,820 with 8952 in patient educational materials. The majority of the words in patient educational materials were present in questions from diabetic patients with $25.06 \%$ (2244/8952) of the words not in questions from diabetic patients (Figure 3).

Table 1. An overview of the two corpora.

\begin{tabular}{llllll}
\hline Corpus & Number & $\begin{array}{l}\text { Total word count } \\
\text { (mapping rate) }\end{array}$ & $\begin{array}{l}\text { Word count, mean } \\
(\text { SD) }\end{array}$ & $\begin{array}{l}\text { Unique word count } \\
\text { Unique UMLS } \\
\text { concepts, } \mathrm{n}\end{array}$ \\
\hline $\begin{array}{l}\text { Questions from diabetic } \\
\text { patients }\end{array}$ & 7510 & $829,893(91.18 \%)$ & $110(36)$ & 41,820 & 19,616 \\
\begin{tabular}{l} 
Patient educational materials \\
\hline
\end{tabular} & 144 & $139,463(93.31 \%)$ & $968(115)$ & 8952 & 7924 \\
\hline
\end{tabular}

\footnotetext{
${ }^{\text {a }}$ Mapping rate was presented the probability of words mapped to the UMLS from the total word count. Difference in mapping rate between the two
} corpa was statistically significant $(P<.001)$.

Table 2 shows the top 20 words for each corpus. The diabetes technology, community, and type 1 and latent autoimmune diabetes of adulthood (LADA) were the most common topics posted by questions from diabetic patient users, and topic 5, topic 3, and topic 8 were the main topics by topic modeling in patient educational materials documents as shown in Table 3. Table 4 shows some examples of topics obtained using topic modeling, which lists the top 20 words and their corresponding weights for each of the topics. The results of the topic vocabulary similarity analysis calculating the cosine similarity between each two topics of the two corpora are presented by a heat map graphic (Figure 4). There was no vocabulary similarity between the questions from diabetic patients categories and the patient educational materials topics, but one topic to one another topic in interior questions from diabetic patients corpus had high linguistic similarity. The semantic group distribution of the two corpora was significantly different (Figure 5) where procedures, phenomena, objects, living beings, disorders, and anatomy were more prevalent in patient educational materials, whereas physiology, genes and molecular sequences, devices, and chemicals and drugs were more prevalent in patient educational materials.

Figure 3. The Venn diagram of the words in the two corpora. There were 35,112 (83.96\%) unique words in the questions from diabetic patients (QDP) corpus and $2244(25.06 \%)$ unique words in the patient educational materials (PEM) corpus.

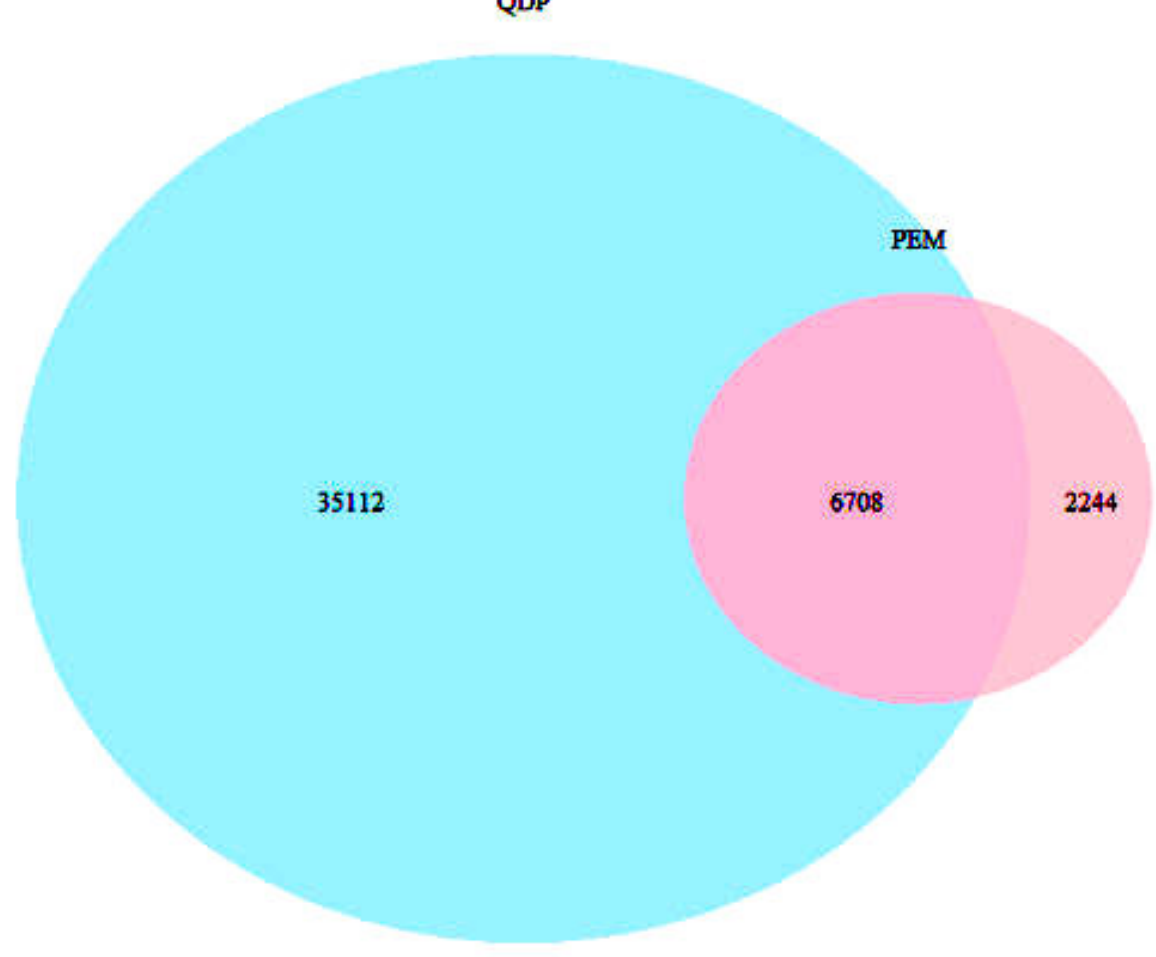


Table 2. The top 20 words in the two corpora.

\begin{tabular}{|c|c|c|c|c|}
\hline \multirow[t]{2}{*}{ Rank } & \multicolumn{2}{|c|}{ Questions from diabetic patients } & \multicolumn{2}{|c|}{ Patient educational materials } \\
\hline & Word & Frequency & Word & Frequency \\
\hline 1 & Diabetes & 9062 & Blood & 3081 \\
\hline 2 & Insulin & 5369 & Insulin & 2504 \\
\hline 3 & Type & 4657 & Glucose & 2074 \\
\hline 4 & Like & 4620 & Diabetes & 1385 \\
\hline 5 & Get & 4457 & Child & 1166 \\
\hline 6 & Time & 4107 & Meal & 1047 \\
\hline 7 & Know & 3875 & Childs & 815 \\
\hline 8 & Pump & 3428 & Care & 801 \\
\hline 9 & Now & 3421 & Health & 797 \\
\hline 10 & Blood & 3388 & Dose & 782 \\
\hline 11 & Day & 3317 & Test & 738 \\
\hline 12 & People & 2789 & Sugar & 728 \\
\hline 13 & First & 2395 & Help & 671 \\
\hline 14 & Sugar & 2383 & Provider & 638 \\
\hline 15 & Go & 2309 & Day & 635 \\
\hline 16 & Back & 2290 & High & 627 \\
\hline 17 & See & 2219 & Evening & 583 \\
\hline 18 & Think & 2148 & Take & 583 \\
\hline 19 & High & 2088 & Time & 571 \\
\hline 20 & Use & 2036 & Eat & 547 \\
\hline
\end{tabular}


Table 3. Category and topic distribution of the two corpora.

\begin{tabular}{|c|c|}
\hline Category/topic ${ }^{\mathrm{a}}$ & $\mathrm{n}(\%)$ \\
\hline \multicolumn{2}{|l|}{ Questions from diabetic patients } \\
\hline Type 2 & $454(6.0)$ \\
\hline Type 1 and LADA & $1609(21.4)$ \\
\hline TuDiabetes website & $97(1.3)$ \\
\hline Treatment & $507(6.8)$ \\
\hline Mental and emotional wellness & $92(1.2)$ \\
\hline Healthy living & $187(2.5)$ \\
\hline Food & $321(4.3)$ \\
\hline Diabetes technology & $1903(25.4)$ \\
\hline Diabetes complications and other conditions & $211(2.8)$ \\
\hline Diabetes and pregnancy & $117(1.6)$ \\
\hline Diabetes advocacy & $253(3.4)$ \\
\hline Community & $1759(23.4)$ \\
\hline \multicolumn{2}{|l|}{ Patient educational materials (PEM) } \\
\hline PEM1 & $6(4.2)$ \\
\hline PEM2 & $5(3.5)$ \\
\hline PEM3 & $13(9.0)$ \\
\hline PEM4 & $6(4.2)$ \\
\hline PEM5 & $15(10.4)$ \\
\hline PEM6 & $10(6.9)$ \\
\hline PEM7 & $3(2.1)$ \\
\hline PEM8 & $11(7.6)$ \\
\hline PEM9 & $5(3.5)$ \\
\hline PEM10 & $7(4.9)$ \\
\hline PEM11 & $9(6.3)$ \\
\hline PEM12 & $9(6.3)$ \\
\hline PEM13 & $6(4.2)$ \\
\hline PEM14 & $8(5.6)$ \\
\hline PEM15 & $3(2.1)$ \\
\hline PEM16 & $3(2.1)$ \\
\hline PEM17 & $6(4.2)$ \\
\hline PEM18 & $7(4.9)$ \\
\hline PEM19 & $5(3.5)$ \\
\hline PEM20 & $7(4.9)$ \\
\hline
\end{tabular}

${ }^{a}$ The categories of the questions from diabetic patients corpus were labeled as the website provided, and the topics of the patient educational material (PEM) corpus were generated using LDA topic modeling. The topic proportion was calculated with the maximum distribution of document. 
Table 4. Sample topics in the patient educational materials (PEM) corpus.

\begin{tabular}{|c|c|c|}
\hline PEM group & Top 20 most prominent words (corresponding weight) & Topic \\
\hline PEM2 & $\begin{array}{l}\text { Disease }(0.071) \text {, kidney }(0.043) \text {, risk }(0.037) \text {, heart }(0.031) \text {, health }(0.023) \text {, pressure }(0.021) \text {, care }(0.018) \text {, } \\
\text { provider }(0.017) \text {, factors }(0.017) \text {, people }(0.017) \text {, kidneys }(0.015) \text {, cholesterol }(0.012) \text {, high }(0.011) \text {, lifestyle } \\
(0.010) \text {, levels }(0.010) \text {, protein }(0.010) \text {, control }(0.009) \text {, body }(0.008) \text {, urine }(0.008) \text {, medications }(0.008)\end{array}$ & Complication-kidney \\
\hline PEM8 & $\begin{array}{l}\text { Food }(0.039) \text {, fruit }(0.024) \text {, cup }(0.022) \text {, foods }(0.022) \text {, eat }(0.020) \text {, sugar }(0.020) \text {, fat }(0.019) \text {, carbohydrate } \\
(0.017) \text {, meal }(0.016) \text {, plan }(0.015) \text {, milk }(0.015) \text {, protein }(0.014) \text {, carbohydrates }(0.013) \text {, snack }(0.013) \text {, vegetables } \\
(0.013) \text {, grams }(0.011) \text {, meals }(0.011) \text {, make }(0.011) \text {, calories }(0.010) \text {, serving }(0.010)\end{array}$ & Food \\
\hline PEM13 & $\begin{array}{l}\text { Care }(0.024) \text {, feet }(0.023) \text {, problems }(0.022) \text {, provider }(0.020) \text {, pain }(0.020) \text {, health }(0.017) \text {, term }(0.017) \text {, } \\
\text { symptoms }(0.015) \text {, peripheral }(0.015) \text {, website }(0.014) \text { nerves }(0.013) \text {, legs }(0.012) \text {, system }(0.012) \text {, neuropathy } \\
(0.012) \text {, stroke }(0.012) \text {, walking }(0.011) \text {, figure }(0.011) \text {, shoes }(0.011) \text {, infections }(0.009) \text {, brain }(0.009)\end{array}$ & Complication-foot \\
\hline
\end{tabular}

Figure 4. Heat map of questions from diabetic patients categories and patient educational materials topics based on cosine similarity of word vectors weighted using TF-IDF or topic word distribution. The clustering is based on Euclidean distance.

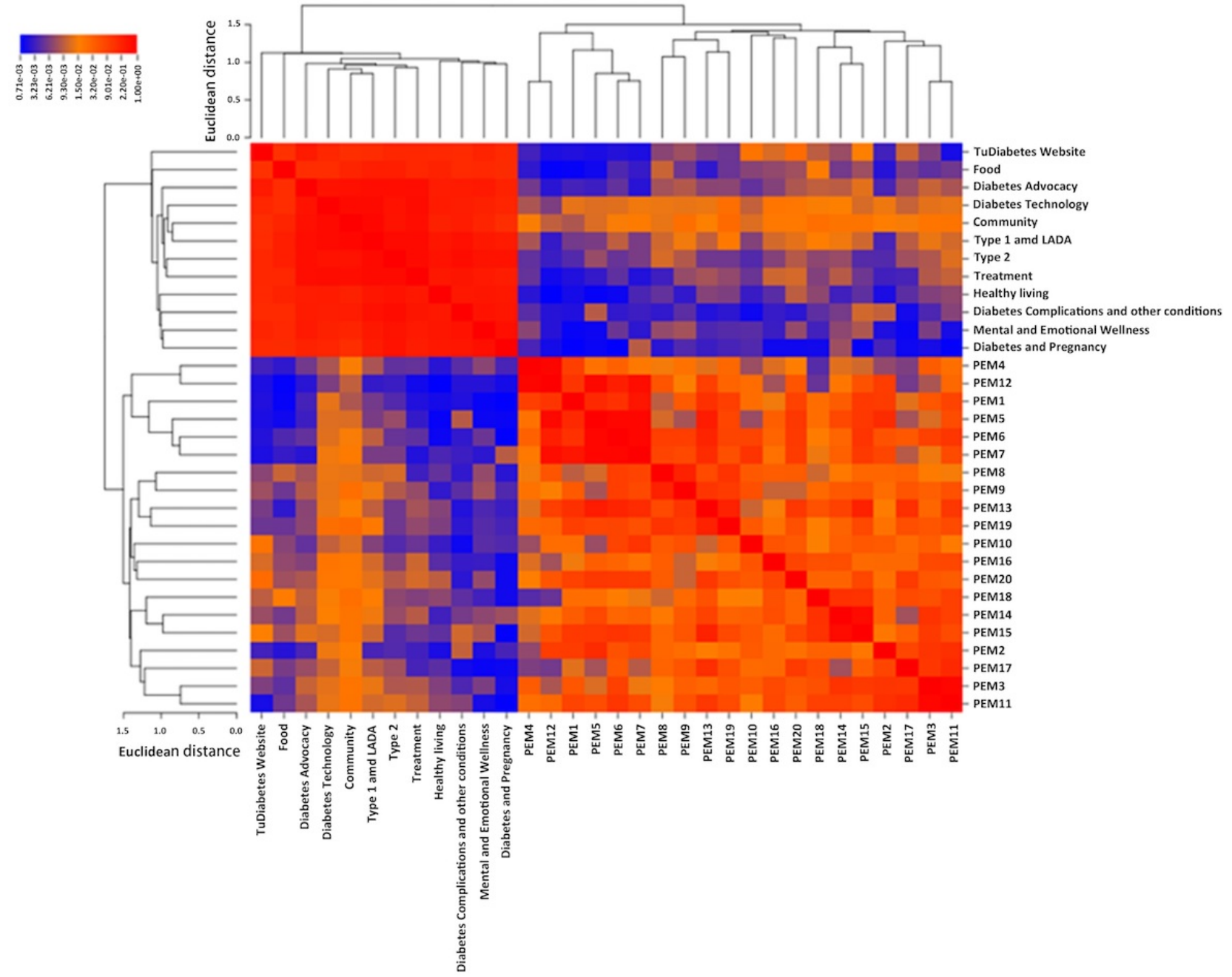


Figure 5. Distribution of 10 clinical semantic groups in the two corpora: questions from diabetic patients (QDP) and patient educational materials (PEM). ANAT: anatomy; CHEM: chemicals and drugs; DEVI: devices; DISO: disorders; GENE: genes and molecular sequences; LIVB: living beings; OBJC: objects; PHEN: phenomena; PHYS: physiology; PROC: procedures.

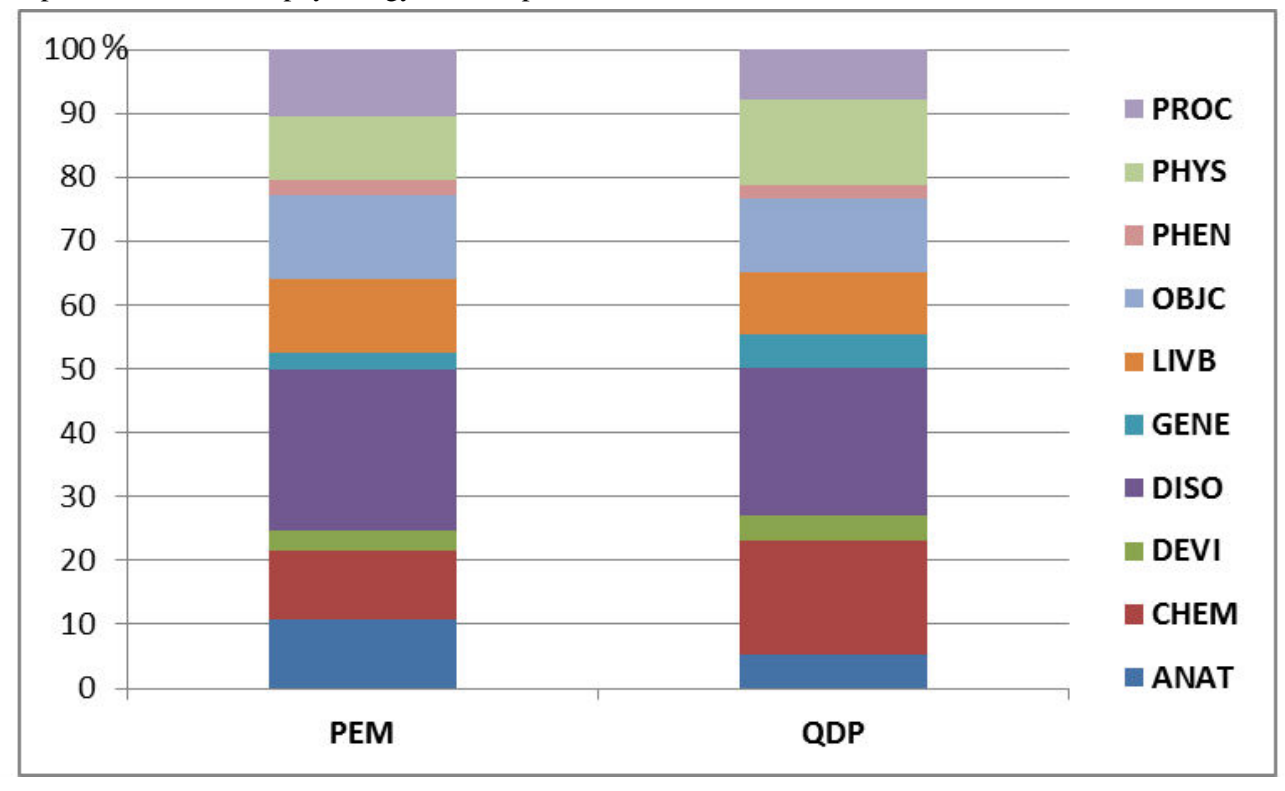

\section{Network Analysis}

Figure 6 shows the networks of topics or semantic groups with questions for those with the topic/semantic group frequency larger than one (ie, question 5220 matched to topic 8 with a topic frequency of 2.22 , and question 4124 matched to the physiology semantic group with semantic group frequency of 4.02). In the network of topic modeling-based model (Figure 6 ), all patient educational materials topics were presented, there were more questions matched to topic 4 , topic 8 , and topic 9 , whereas some topics (eg, topic 1, topic 2, topic 3, or topic 10) were relevant to a small number of questions. Some questions were associated with very specific topics. For example, question 6722 from the diabetes complication and other condition topic in questions from diabetic patients corpus, the content of the question was: "Do you have neuropathy? Introduce yourself here! Foot pain, numbness, nerve pain, does anyone else know what I'm going through? Yes, we do!" It had the unique matching to the PEM13 topic (ie, complication-foot topic). In the network of semantic group-based model (Figure 6), the objects, physiology, and live beings groups had more questions. Similarly, some questions were associated with very specific semantic groups. For example, question 7113 from the diabetes technology topic in the questions from diabetic patients corpus, the content of the question was: "Are you an Accu-Chek user?
Jump in here For users of ACCU-CHEK glucose meters." It was mapped to the devices semantic group. The combination of the two networks (Figure 6) showed that for some questions the two models, topic modeling-based and semantic group-based, were complementary to each other. For example, question 2760 belonged to the diabetes complication and other condition topic in the questions from diabetic patients corpus, the content of the question was: "Balance neuropathy I don't have the tingling, numbness, painful neuropathy, but the feelings I have in my feet somehow aren't being delivered to my balance center. I am having a nerve conduction test an electromyography. Any advice?" It is relevant to the complication-foot topic (ie, PEM13) and also to the disorders semantic group.

\section{Performance Comparison}

The two experts had a high level of agreement in relevance judgment $(\kappa=0.90)$. The performance of the three models is presented in Figure 7 and Table 5. The topic modeling-based model outperformed the other two models at each rank, and the semantic group-based model had a better performance than the baseline VSM model. For example, for the top-retrieved document, the precision of the topic modeling-based, semantic group-based, and VSM models were 0.670 (67.0\%), 0.628 (62.8\%), and $0.543(54.3 \%)$, respectively. 
Figure 6. (A) Network formed using the topic modeling-based model (TMB) with topic frequency cutoff 1, (B) network formed based on the semantic group-based model (SGB) with semantic group frequency cutoff 1, and (C) a combined network by linking the two networks (TMB+SGB) based on questions.

\section{(A) TMB}

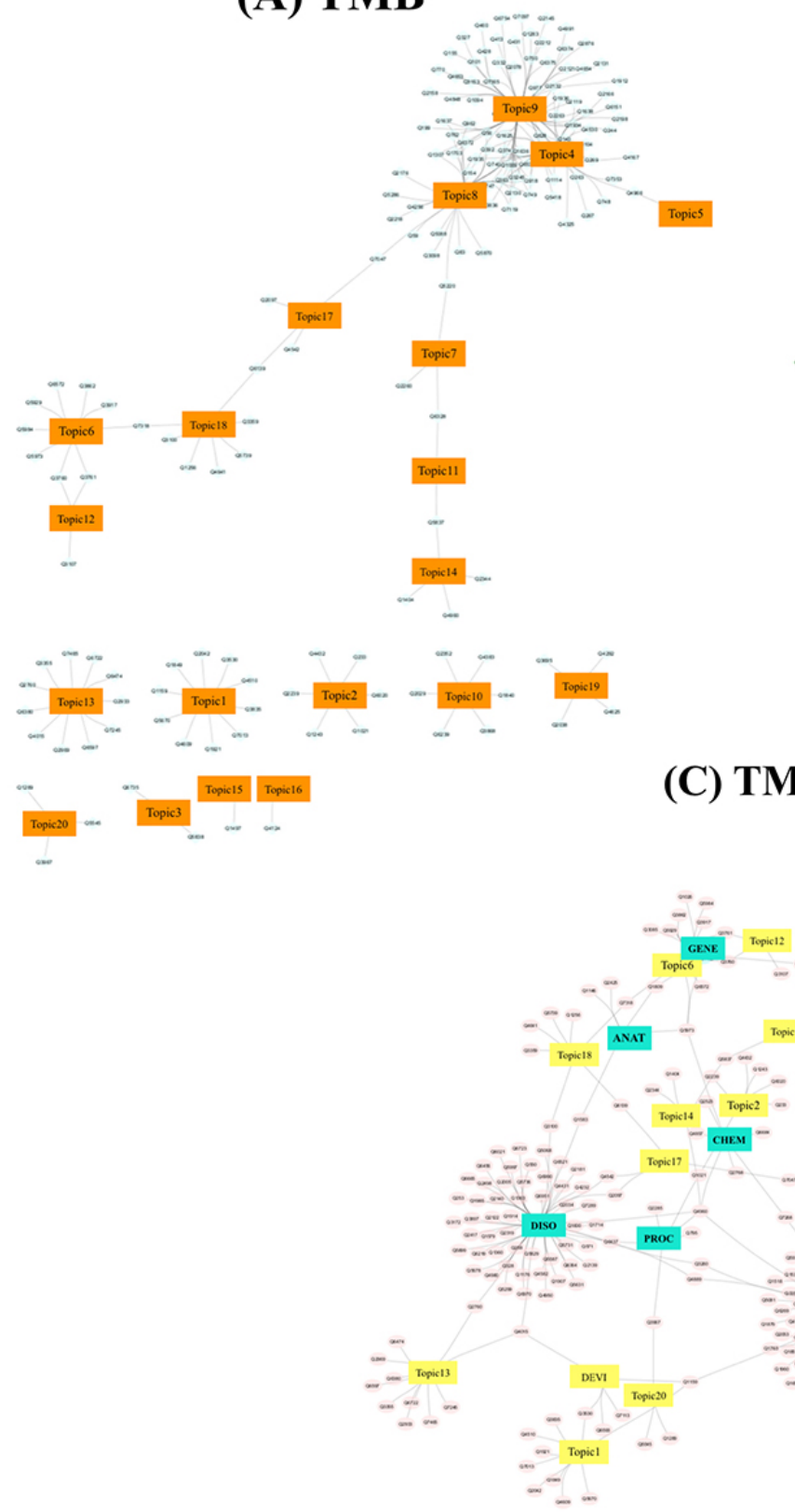

\section{(B) SGB}

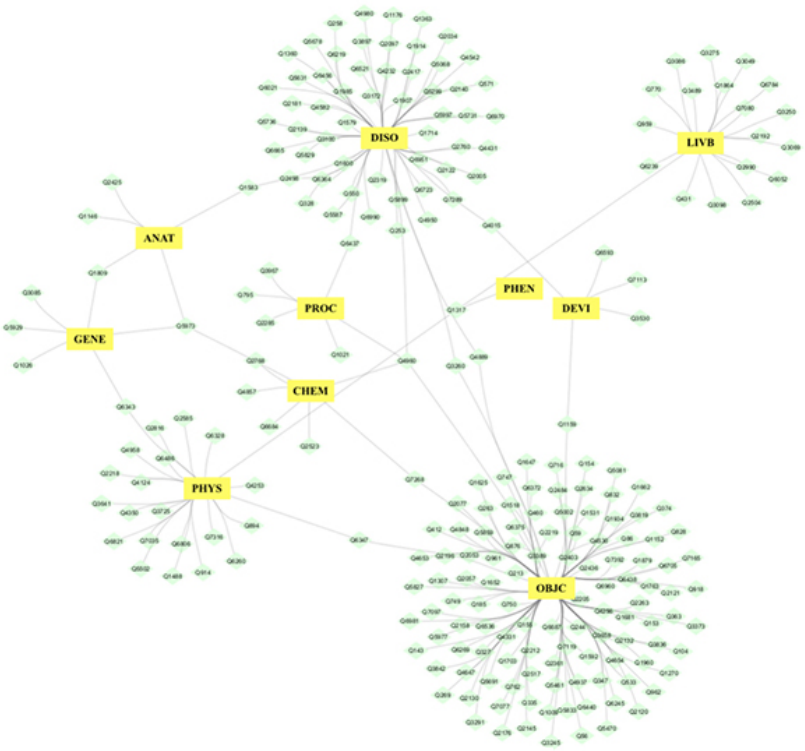

Table 5. Performance comparison of topic modeling-based, semantic group-based, and vector space model (VSM) models.

\begin{tabular}{llllllll}
\hline Model & \multicolumn{2}{l}{ Mean precision } & & & & & \\
& P 1 & P 2 & P 3 & P 4 10 & P 5 & P 20 \\
\hline Topic modeling-based & 0.670 & 0.622 & 0.596 & 0.588 & 0.596 & 0.579 & 0.572 \\
Semantic group-based & 0.628 & 0.606 & 0.585 & 0.582 & 0.581 & 0.564 & 0.547 \\
VSM & 0.543 & 0.532 & 0.532 & 0.529 & 0.528 & 0.528 & 0.531 \\
\hline
\end{tabular}


Figure 7. Precision at rank 1 to 20 for topic modeling-based (TMB), semantic group-based (SGB), and vector space model (VSM) models.

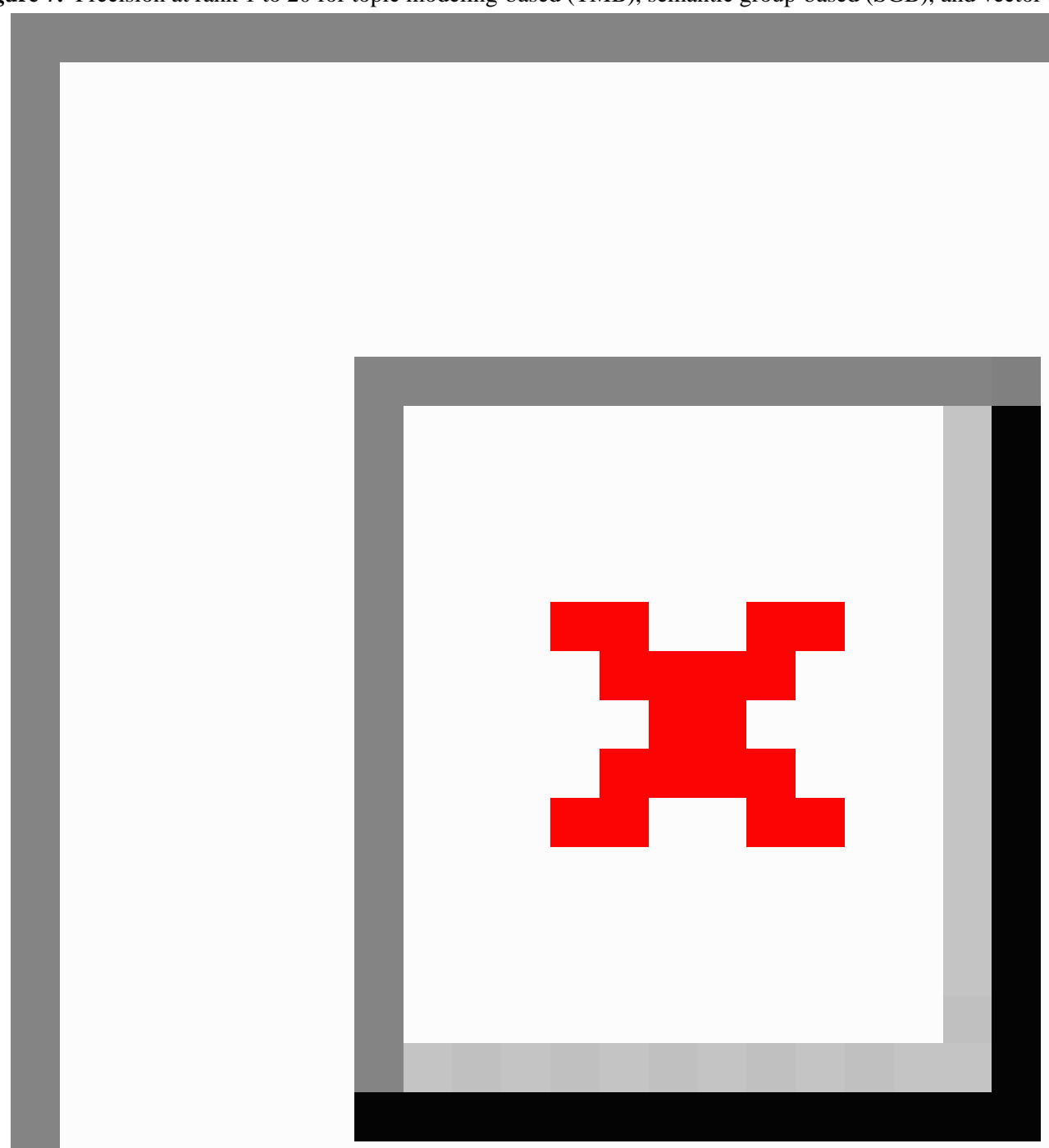

\section{Discussion}

In summary, we investigated the use of the state-of-the-art information retrieval approaches to recommend diabetes education materials for questions available in an online forum for diabetes by leveraging a corpus assembled from diabetes education materials and a corpus assembled from an online forum. Our study shows that the language used in patient education materials is different from the language used in questions from an online forum. A topic modeling-based model has the potential to accurately recommend patient education material to a given question. Both topic modeling-based and semantic group-based models outperform the baseline VSM model. Network analysis illustrates that the network formed by topic modeling and the network formed by semantic groups are different and the combination of them may yield a better strategy.

Literature has shown that the language used by patients is different from the one used by clinicians [27]. Our study demonstrated that there is a language difference between patient education materials and questions in an online forum even though the target audiences of patient education materials are the patients. Patient educational materials are often produced internally by hospital staff without sufficient consideration of the patients intended to use them [28]. In our study, patient education materials tend to cover clinical and patient life topics, whereas patients tend to ask about disease-specific technology and treatment from the top words in Table 2. In addition, the 
semantic group of questions from diabetic patients corpus included mainly chemical drugs, physiology, devices, and gene aspects more than patient educational materials corpus, and these semantic groups also related to complication, treatment, and technology categories. There was consistency between the primary category distribution of questions from diabetic patients and their semantic groups. Therefore, analyzing online forums can identify information needs of patients and provide an opportunity to create patient-centric education materials.

The study demonstrated that topic modeling can mitigate the vocabulary difference between two corpora and achieve the best performance in recommending education materials to questions. In Figure 7, we found that the topic modeling-based model outperformed the other two models. Through topic modeling, topics and their probability distribution can be calculated for analyzing document similarity, which has been explored for document classification and personalized recommendation. For example, the iDoctor used LDA topic modeling for personalized and professionalized medical recommendation based on data available at crowd-sourced review websites [29] and Kandula et al's [18] study also showed that the LDA topic modeling can better recommend patient education material to diabetic patients based on clinical notes. Our network analysis demonstrates that the topic modeling-based and semantic group-based models form two independent networks, which may imply that combining the two automated models has the potential to improve the recommendation.

Here, we only studied one disease and used our institutional patient education materials. More research is needed to see if our findings can be generalized. One direction for future work is to extend our study to other disease areas, other patient education material resources, and online forums.

\section{Acknowledgments}

The study was supported by National Institute of Health research grants, R01LM011934-01A1 and R01EB019403 and grant NO.2014B010118005 from Guangdong Provincial Department of Science and Technology, Guangzhou, Guangdong, China. The first author was funded by China Scholarship Council.

\section{Conflicts of Interest}

None declared.

\section{Multimedia Appendix 1}

Information retrieval algorithms.

[PDF File (Adobe PDF File), 128KB-Multimedia Appendix 1]

\section{References}

1. IDF Diabetes Atlas. URL: http://www.diabetesatlas.org/ [accessed 2017-09-27] [WebCite Cache ID 6tnpY8Vwj]

2. Zhuo X, Zhang P, Barker L, Albright A, Thompson TJ, Gregg E. The lifetime cost of diabetes and its implications for diabetes prevention. Diabetes Care 2014 Sep;37(9):2557-2564. [doi: 10.2337/dc13-2484] [Medline: 25147254]

3. Heinrich E, Schaper N, de Vries N. Self-management interventions for type 2 diabetes: a systematic review. Eur Diabetes Nurs 2015 Feb 17;7(2):71-76 [FREE Full text] [doi: 10.1002/edn.160]

4. Moser A, van der Bruggen H, Widdershoven G, Spreeuwenberg C. Self-management of type 2 diabetes mellitus: a qualitative investigation from the perspective of participants in a nurse-led, shared-care programme in the Netherlands. BMC Public Health 2008 Mar 18;8:91 [FREE Full text] [doi: 10.1186/1471-2458-8-91] [Medline: 18366665]

5. Devi R, Kapoor B, Singh M. Effectiveness of self-learning module on the knowledge and practices regarding foot care among type II diabetes patients in East Delhi, India. Int J Community Med Pub Health 2017;3(8):2133-2141. [doi: 10.18203/2394-6040.ijcmph20162559]

6. Kanthawala S, Vermeesch A, Given B, Huh J. Answers to health questions: Internet search results versus online health community responses. J Med Internet Res 2016 Apr 28;18(4):e95 [FREE Full text] [doi: 10.2196/jmir.5369] [Medline: 27125622]

7. Zheng Y, Wu L, Su Z, Zhou Q. Development of a diabetes education program based on modified AADE diabetes education curriculum. Int J Clin Exp Med 2014;7(3):758-763 [FREE Full text] [Medline: 24753774]

8. Hermanns N, Kulzer B, Ehrmann D, Bergis-Jurgan N, Haak T. The effect of a diabetes education programme (PRIMAS) for people with type 1 diabetes: results of a randomized trial. Diabetes Res Clin Pract 2013 Dec;102(3):149-157. [doi: 10.1016/j.diabres.2013.10.009] [Medline: 24210673]

9. Haas L, Maryniuk M, Beck J, Cox CE, Duker P, Edwards L, 2012 Standards Revision Task Force. National standards for diabetes self-management education and support. Diabetes Care 2014 Jan;37 Suppl 1:S144-S153 [FREE Full text] [doi: 10.2337/dc14-S144] [Medline: 24357210]

10. Baeza-Yates R, Ribeiro-Neto B. Modern Information Retrieval. New York: ACM Press; 1999.

11. Raghavan V, Wong S. A critical analysis of vector space model for information retrieval. J Am Soc Inform Sci 1986;37(5):279.

12. Salton G, McGill M. Introduction to Modern Information Retrieval. New York: McGraw-Hill; 1983. 
13. Miller G. WordNet: a lexical database for English. Commun ACM 1995;38(11):39-41. [doi: 10.1145/219717.219748]

14. Wang C, Blei D. Collaborative topic modeling for recommending scientific articles. In: Proceedings of the 17th ACM SIGKDD International Conference on Knowledge Discovery and Data Mining. 2011 Aug Presented at: KDD '11 17th ACM SIGKDD International Conference on Knowledge Discovery and Data Mining; Aug 21-24, 2011; San Diego, CA p. $448-456$ URL: https://doi.org/10.1145/2020408.2020480

15. Yi X, Allan J. A comparative study of utilizing topic models for information retrieval. In: ECIR 2009: Advances in Information Retrieval. New York: Springer; 2009:29-41.

16. Gardner M. Information retrieval for patient care. BMJ 1997 Mar 29;314(7085):950-950 [FREE Full text] [doi: 10.1136/bmj.314.7085.950]

17. Ebell MH, Barry HC. InfoRetriever: rapid access to evidence-based information on a hand-held computer. MD Comput 1998;15(5):289-297. [Medline: 9753974]

18. Kandula S, Curtis D, Hill B, Zeng-Treitler Q. Use of topic modeling for recommending relevant education material to diabetic patients. AMIA Annu Symp Proc 2011;2011:674-682 [FREE Full text] [Medline: 22195123]

19. Apache Tika. URL: https://tika.apache.org/ [accessed 2017-09-27] [WebCite Cache ID 6tnsHd7mi]

20. tudiabetes.org. URL: http://www.tudiabetes.org/forum/ [accessed 2017-09-27] [WebCite Cache ID 6tnsM3ZQi]

21. Bodenreider O. The Unified Medical Language System (UMLS): integrating biomedical terminology. Nucleic Acids Res 2004 Jan 1;32(Database issue):D267-D270 [FRE Full text] [doi: 10.1093/nar/gkh061] [Medline: 14681409]

22. Phan X, Nguyen L, Horiguchi S. Learning to classify shortsparse text \&amp;amp; web with hidden topics from large-scale data collections. In: Proceedings of the 17th International Conference on World Wide Web. 2008 Apr Presented at: WWW 2008 7th International Conference on World Wide Web; Apr 21-25, 2008; Beijing, China p. 91-100 URL: https://doi.org/ $\underline{10.1145 / 1367497.1367510}$

23. Blei D, Ng A, Jordan M. Latent Dirichlet Allocation. J Mach Learn Res 2003;3:993-1022.

24. R Core Team. R Language Definition. Vienna, Austria: R foundation for statistical computing; 2000.

25. Shannon P, Markiel A, Ozier O, Baliga NS, Wang JT, Ramage D, et al. Cytoscape: a software environment for integrated models of biomolecular interaction networks. Genome Res 2003 Nov;13(11):2498-2504 [FREE Full text] [doi: $\underline{10.1101 / g r .1239303}$ ] [Medline: 14597658$]$

26. Pedregosa F, Varoquaux G, Gramfort A, Michel V, Thirion B, Grisel O, et al. Scikit-learn: machine learning in Python. J Mach Learn Res 2011;12:2825-2830.

27. Zeng Q, Kogan S, Ash N, Greenes RA. Patient and clinician vocabulary: how different are they? Stud Health Technol Inform 2001;84(Pt 1):399-403. [Medline: 11604772]

28. Payne S, Large S, Jarrett N, Turner P. Written information given to patients and families by palliative care units: a national survey. Lancet 2000 May 20;355(9217):1792 [FREE Full text] [doi: 10.1016/S0140-6736(00)02272-8] [Medline: 10832835]

29. Zhang Y, Chen M, Huang D, Wu D, Li Y. iDoctor: personalized and professionalized medical recommendations based on hybrid matrix factorization. Future Gener Comp Sy 2017 Jan;66:30-35 [FREE Full text] [doi: 10.1016/j.future.2015.12.001]

\section{Abbreviations \\ LADA: latent autoimmune diabetes of adulthood \\ LDA: Latent Dirichlet Allocation \\ UMLS: Unified Medical Language System \\ VSM: vector space model}

Edited by G Eysenbach; submitted 24.03.17; peer-reviewed by E Da Silva, K Fitzner; comments to author 15.06.17; revised version
received 06.08.17; accepted 29.08.17; published 16.10.17
Please cite as:
Zeng Y, Liu X, Wang Y, Shen F, Liu S, Rastegar-Mojarad M, Wang L, Liu H
Recommending Education Materials for Diabetic Questions Using Information Retrieval Approaches
J Med Internet Res 2017;19(10):e342
URL: $\underline{\text { http://www.jmir.org/2017/10/e342/ }}$
doi: $\underline{10.2196 / \text { jmir.7754 }}$
PMID: $\underline{29038097}$

(C) Yuqun Zeng, Xusheng Liu, Yanshan Wang, Feichen Shen, Sijia Liu, Majid Rastegar Mojarad, Liwei Wang, Hongfang Liu. Originally published in the Journal of Medical Internet Research (http://www.jmir.org), 16.10.2017. This is an open-access article distributed under the terms of the Creative Commons Attribution License (https://creativecommons.org/licenses/by/4.0/), which permits unrestricted use, distribution, and reproduction in any medium, provided the original work, first published in the Journal 
of Medical Internet Research, is properly cited. The complete bibliographic information, a link to the original publication on http://www.jmir.org/, as well as this copyright and license information must be included. 